\title{
Processo de Gestão: Uma proposta de modelo para estratégia nas empresas na busca por obtenção de vantagem competitiva organizacional
}

\author{
Management Process: A proposed model for strategy in companies seeking to obtain organizational \\ competitive advantage
}

Proceso de Gestión: Propuesta de modelo de estrategia en empresas que buscan obtener ventaja competitiva organizacional

Recebido: 19/07/2021 | Revisado: 22/07/2021 | Aceito: 23/07/2021 | Publicado: 26/07/2021

\author{
Marcos de Oliveira Morais \\ ORCID: https://orcid.org/0000-0002-5981-4725 \\ Universidade Estácio de Sá, Brasil \\ Universidade Santo Amaro, Brasil \\ E-mail: marcostecnologia2001@gmail.com \\ Hernani Vidigal \\ ORCID: https://orcid.org/0000-0002-8924-8859 \\ Fundação Instituto de Administração, Brasil \\ E-mail: hpvidigal@gmail.com
}

\begin{abstract}
Resumo
O processo inovativo passa a ser essencial para as organizações, pois, agrega valor a produtos, processos e serviços mantendo a empresa no mercado e gerando vantagem competitiva, ciente disso as empresas buscam gerir cada vez mais novas ideias. Nesse contexto, a geração e gestão de ideias torna-se um fator necessário para o fomento da gestão organizacional em meio as altas competitividades e mercados cada vez mais acirrados. Este artigo objetiva apresentar o desenvolvimento de um modelo para a ampliação da vantagem competitiva organizacional que possibilite auxiliar e agregar valor as decisões nas organizações. Foi utilizada metodologia de referências bibliográficas em face a nova proposta. Foram utilizados também elementos gerenciais já existentes para dar base a esta nova proposta. A busca por inserção de novas ferramentas gerenciais passa a ser de extrema relevância no crescimento organizacional independentemente da organização a ser estudada ou aplicada, onde a busca pelo crescimento das pessoas envolvidas também deve ser levado em consideração estimulando as questões socio-ambientais.
\end{abstract}

Palavras-chave: Inovação; Gestão do conhecimento; Estratégia; Competitividade.

\begin{abstract}
The innovative process becomes essential for organizations, as it adds value to products, processes and services, keeping the company in the market and generating competitive advantage, aware that companies seek to manage more and more new ideas. In this context, the generation and management of ideas becomes a necessary factor for the promotion of organizational management amid high competitiveness and increasingly fierce markets. This article aims to present the development of a model for the expansion of organizational competitive advantage that makes it possible to assist and add value to decisions in organizations. Bibliographic references methodology was used in view of the new proposal. Existing managerial elements were also used to base this new proposal. The search for insertion of new management tools becomes extremely relevant in organizational growth regardless of the organization to be studied or applied, where the search for the growth of the people involved must also be taken into account, stimulating socio-environmental issues. Keywords: Innovation; Knowledge management; Strategy; Competitiveness.

\section{Resumen}

El proceso innovador se vuelve fundamental para las organizaciones, ya que agrega valor a los productos, procesos y servicios, manteniendo a la empresa en el mercado y generando ventaja competitiva, conscientes de que las empresas buscan gestionar cada vez más ideas nuevas. En este contexto, la generación y gestión de ideas se convierte en un factor necesario para el impulso de la gestión organizacional en un entorno de alta competitividad y mercados cada vez más feroces. Este artículo tiene como objetivo presentar el desarrollo de un modelo para la expansión de la ventaja competitiva organizacional que permita asistir y agregar valor a las decisiones en las organizaciones. Se utilizó la metodología de referencias bibliográficas a la vista de la nueva propuesta. También se utilizaron elementos de gestión existentes para basar esta nueva propuesta. La búsqueda de inserción de nuevas herramientas de gestión adquiere suma relevancia en el crecimiento organizacional independientemente de la organización a estudiar o aplicar, donde también se debe tener en cuenta la búsqueda del crecimiento de las personas involucradas, estimulando temas socioambientales. Palabras clave: Innovación; Gestión del conocimiento; Estrategia; Competitividad.
\end{abstract}




\section{Introdução}

Nas últimas duas décadas, com o avanço das novas tecnologias a utilização de novos sistemas de informações tornaram as ações gerenciais nas organizações cada vez mais dinãmicas sejam estas sobre pressões internas ou de mercado e isso inclui a presença constante de novos competidores em todo o mundo forçando a ter uma maior concorrencia (Zangoueinezhad e Moshabaki, 2009).

Segundo Miller (2002), as informações devem ser sistemáticas de modo a questionar e desafiar a estratégia da organização, na perspectiva de fornecer informações rápidas e seguras, reduzindo incertezas e riscos nas tomadas de decisões. Silva et al. (2013) revelaram que, em decorrência das mudanças tecnológicas, as empresas estão mais conscientes da importância do processo de inovação e estão investindo mais em melhorias de processos e produtos. Souza e Bruno-Faria (2013) apontaram que a capacidade de inovar é um atributo que passa a ser crucial no contexto organizacional e que o processo de inovação pode gerar vantagem competitiva para as organizações.

Muitas empresas ainda não dispõem de processo de gestão estruturados e empregam diferentes ferramentas/metodologias de gestão, e em alguns casos têm problemas na forma sequencial ou lógica intuitiva com que realizam as atividades gerenciais (De Oliveira, 2010). Para que as empresas possam operar em ambientes cada vez mais competitivos, elas precisam determinar sua capacidade de inovação organizacional que está relacionanda com multicaracterísticas organizacionais com destaque na atuação de competências diferenciadas, gestão da alocação de recursos, controle de processos e metodologias (Greening; Turban, 2000; Tidd et al., 2007).

De acordo com Karlsson (2010), o manuseio de ideias nas organizações envolve uma série de desafios. Nesse sentido Karlsson afirma que é necessário que haja algumas alternativa como ponto central para reunir todas as ideias. Outro desafio mencionado é que para um gerenciamento saudável de ideias não basta apenas uma solução de Tecnologia da Informação é necessária a adoção de uma infraestrutura com diretrizes e processos integrados entre pessoas e a inovação, bem como o alinhamento com a cultura organizacional da empresa.

Portanto, este artigo objetiva apresentar o desenvolvimento de uma proposta de modelo para a criação de vantagem competitiva organizacional utilizado conceitos teóricos com vistas a possíveis aplicações práticas no futuro. presente trabalho encontra-se estruturado nas seguintes seções: a introdução que aborda a contextualização do tema; a segunda seção descreve as referências bibliográficas utilizadas, a terceira seção apresenta a metodologia utilizada para o desenvolvimento; a quarta seção apresenta os resultados e discussões; a quinta seção refere-se a conclusão do artigo.

\section{Referencial Teórico}

\subsection{Gestão do Conhecimento Organizacional}

O conhecimento é considerado um recurso estratégico, e, como vários outros recursos, dos quais as organizações dependem, pode ser adquirido. Em contrapartida, a inteligência é mais fugidia, por ser uma característica humana e não tangível o que torna cada indivíduo único (Ramos et, al. 2020). Além disso, o conhecimento pode ser categorizado de duas maneiras, em explícito e implícito (ou tácito) (Polanyi 1966).

Para Nonaka e Takeuchi (1995) estes observam que o explícito está disponível em forma de arquivos, coleções de bibliotecas ou bancos de dados, enquanto alguns tipos de conhecimento tácito são de difícil acesso, por se tratar de experiências acumuladas, criatividade e habilidades que residem nos indivíduos como algo que foi adquirido com o passar do tempo, caracterizado por suas experiências.

Segundo Jannuzzi et al. (2016) os componentes tácito (escasso e de difícil apropriação) e explícito (não escasso e facilmente replicável) do conhecimento não são distintos e exclusivos. Assim, quando criado em um contexto organizacional, o conhecimento está inicialmente "preso" aos indivíduos devido ao seu componente tácito. 
Como uma ferramenta que busca identificar e alinhar o conhecimento coletivo em uma organização, surgiu a Gestão do Conhecimento, tentando melhorar a sua competitividade, juntamente com sua capacidade de inovar e de responder às mudanças (Alavi \& Leidner, 2001). Sua definição, segundo alguns autores, ainda é incompleta, por causa das múltiplas interpretações possíveis. A GC é uma disciplina nova e está evoluindo constantemente (Chuang et al., 2013). Não há uma definição única universalmente aceita, no momento, para a GC (Okumus, 2013).

Probst et al. (2002) propicia a conversão dos problemas organizacionais reais em problemas de conhecimento, demonstrando sua aplicabilidade por meio de ferramentas de avaliação dos efeitos das tomadas de decisões sobre os ativos intelectuais. O ponto forte deste método está na apresentação de várias ferramentas que apoiam a arquitetura de todos os elementos construtivos do conhecimento. A Figura 1 apresenta estes conceitos.

Figura 1: Elementos construtivos da gestão do conhecimento.

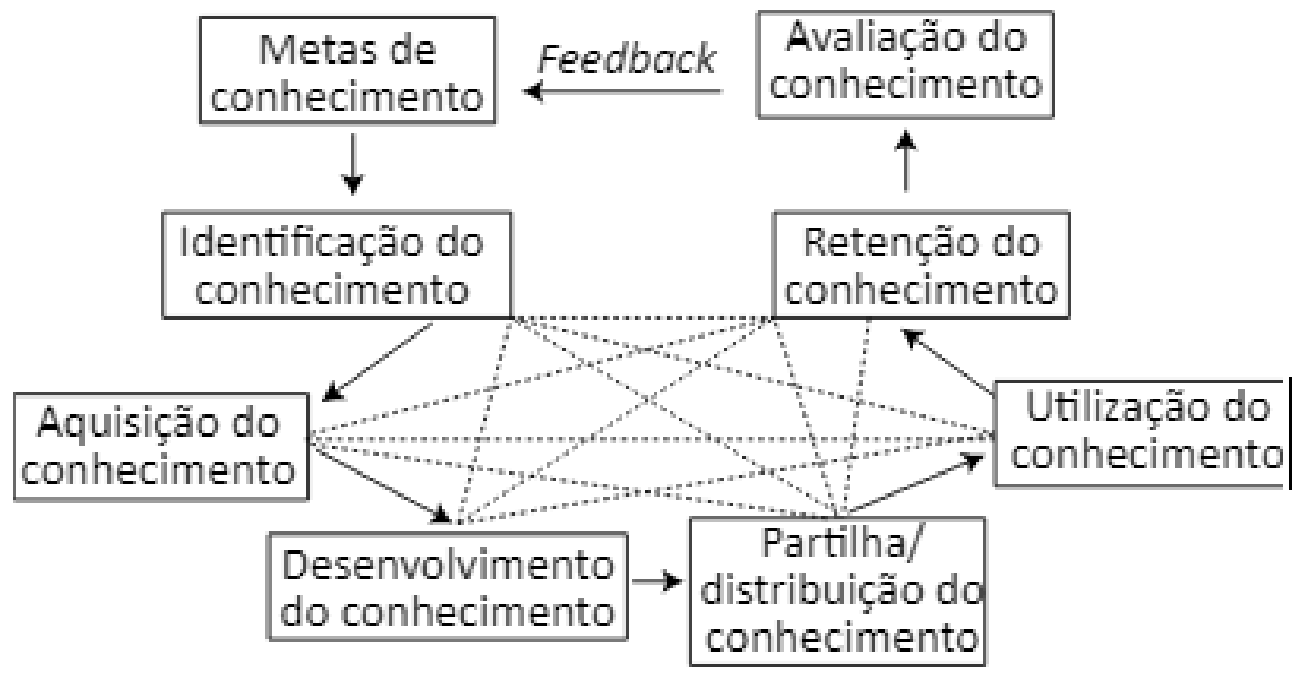

Fonte: Probst et al (2002).

Com base no modelo apresentado e proposto por Probst et al. (2002), percebe-se que os processos envolvidos na definição das metas são o ponto de referência e de partida para uma gestão do conhecimento. Estas metas de conhecimento, assim como quaisquer metas estabelecidas pela organização, definem as diretrizes a serem seguidas por todas as áreas criando uma interface organizacional.

O conhecimento é obtido por meio da reunião de dados, analisados criticamente, e com embasamento, fato que faz com que sejam convertidos em informação e consequentemente conhecimento, que por sua vez, vem imbuído de experiência, valores, contextualização e compreensão (De Sa, Ferreira et al., 2013).

As mudanças no mercado de trabalho e nas organizações com vistas à indústria 4.0, promovem uma grande necessidade de manutenção do conhecimento, sendo este um fato gerador de vantagem competitiva, em outras palavras, favorece a gestão do conhecimento. (Neves, et al.,2018).

Buscar novas formas de criar e agregar valor as empresas por meio do conhecimento passa a ser um importante fator competitivo a ser considerado, onde os profissionais perseguirão, e deverão possuir alto valor agregado intelectual, e as companhias, por sua vez, um grande desafio em manter a sua base de conhecimento sólida o suficiente para manter sua capacidade competitiva. (De Abreu, 2018).

De acordo com Abubakar et al. (2017), a gestão do conhecimento aborda vários outros elementos como recursos humanos, cultura organizacional e estrutura organizacional. Ainda para este autor, os modelos de gerenciamento de 
conhecimento propõem uma interface que se incluam facilitadores, que são mecanismos utilizados pelas instituições para promover o uso consistente do conhecimento gerando novas possibilidades, sejam para a organização como um todo ou mesmo para o indivíduo na sociedade.

\subsection{Inovação Organizacional}

Muitas vezes o tema inovação está associado a algumas variáveis que pretendem explicar ou até mesmo avaliar seus impactos nas organizações, sejam estes referentes a pesquisa e desenvolvimento, a produtividade e principalmente ao conhecimento (Fagerberg \& Sappraset, 2011). Partindo deste princípio a gestão da inovação passa a contribuir com a eficiência e eficácia das organizações, potencializando não somente ganhos financeiros, mas também os ganhos intelectuais (Carrincazeaux \& Gaschet, 2015; Mothe \& Thi, 2010; Schubert, 2010).

A "cultura da inovação organizacional é um fenômeno dinâmico que nos cerca em todas as horas, sendoconstantemente desempenhada e criada por nossas interações com outros". Ainda segundo o mesmo autor também afirma que "oaspecto mais intrigante da cultura como conceito é que ela nos aponta os fenômenos que estão abaixo da superfície,que são poderosos em seu impacto quase invisíveis e comportam um grau considerável de inconsciência” (Schein,2017).

Para Tidd e Bessant (2015), a inovação é orientada pela habilidade de fazer relações, de visualizar oportunidades e de tirar vantagem competitiva destas. A capacidade de distinguir as oportunidades e de criar novas formas de ampliae e explorá-las é indispensável ao processo de gestão da inovação e envolve algumas possibilidades inteiramente novas ao seu processo, como a exploração de avanços tecnológicos totalmente radicais. Os processos de inovação aparecem sob a forma de ações de gestão de inovação tecnológica e performance bem como de práticas de gestão do conhecimento e operações de mudança organizacional (Boly, 2014).

Conforme apresentado por Davila et al., (2018), a inovação tona-se um fenômeno que contempla algumas iniciativas individuais e/ou coletivas de criação de valor para os seus usuários e também de mudanças organizacionais, é considerada elemento potencial para a geração de vantagens competitivas e construção do desenvolvimento econômico organizacional. Embora os conceitos sobre os ciclos econômicos, expressados pelo economista

Schumpeter (1982), são relevantes para a atual ciência econômica possibilitando assim um processo de expansão, potencializando para que se tenha o surgimento de alguma inovação, seja ela incremental ou radical nas empresas que buscam utilizar destas práticas.

Empresas de pequeno e médio porte sentem ainda mais dificuldade para adequar-se à Indústria 4.0, pela complexidade das atividades envolvidas e pela necessidade de investimentos em todas as áreas da empresa. A Indústria 4.0 necessita deinvestimentos de modo que empresas que desejam ajustar-se a esse modelo nem sempre conseguem alcançar os resultados esperados em curto prazo (Souza e Gasparetto, 2018).

A organização deve aplicar seus recursos em áreas estratégicas que proporcionam maior retorno sobre o investimento, do contrário pode afetar a sua capacidade de competir no mercado. A decisão de qual estratégia de inovação melhor se adapta à situação da concorrência externa e do mercado e às condições internas da empresa constitui responsabilidade da equipe de altos executivos, e em última análise é tarefa do diretor geral (Davila et al., 2012).

A cultura para a inovação organizacional permite à empresa ser mais flexível e a se reorganizar com mais agilidade caso haja uma adversidade, uma vez que as pessoas estão envolvidas nos processos independente de sua posição hierárquica na organização.

Segundo Terra (2012), existe uma busca veloz por diferenciação no mercado mundial, que se fazem necessárias estruturas flexíveis que favoreçam as competências globais e aprimorem as criações de forma personalizada as necessidades dos clientes. 
A Figura 2 exemplifica os fatores que envolvem a cultura da inovação relacionados ao processo organizacional.

Figura 2: Cultura da Inovação.

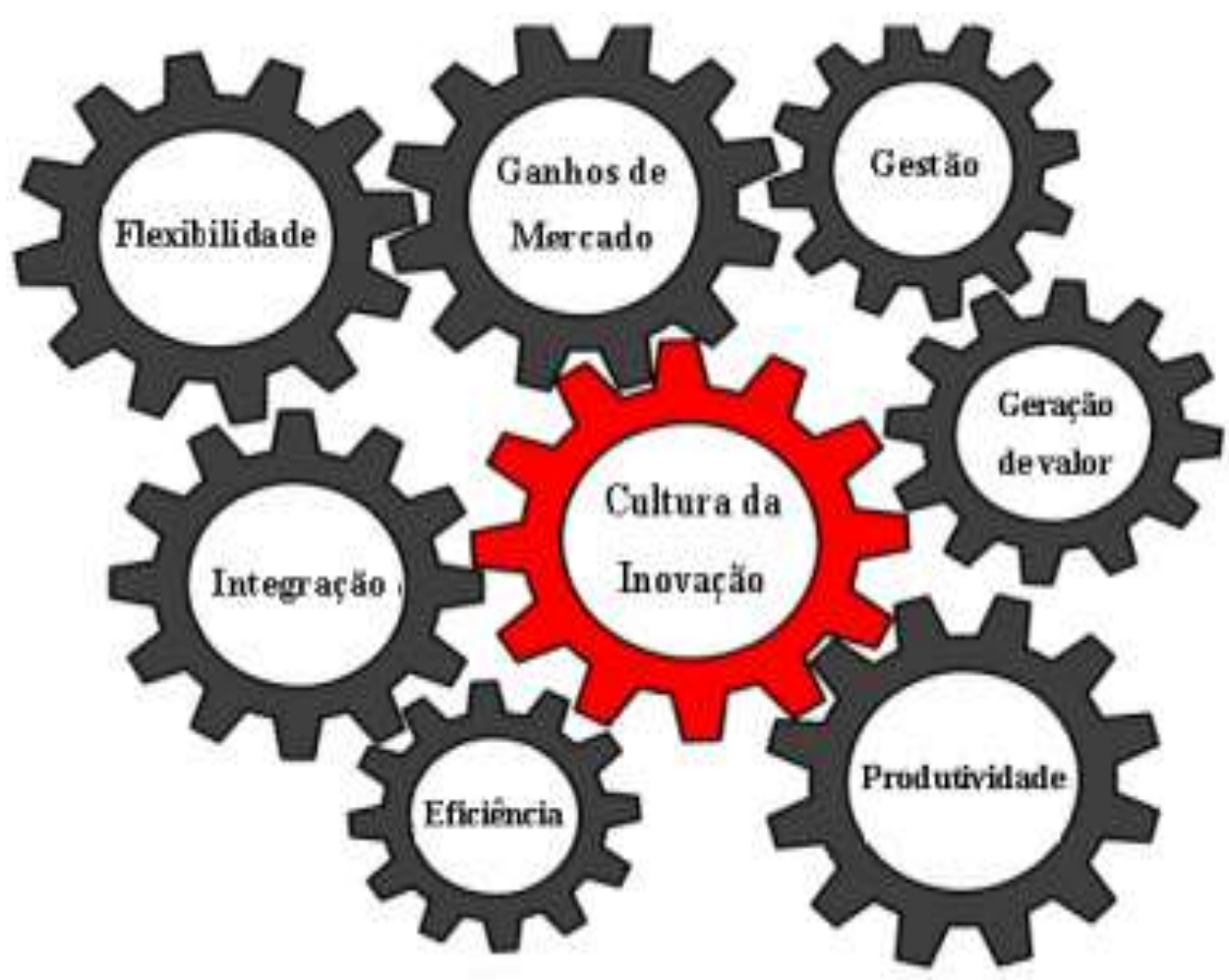

Fonte: de Oliveira Morais (2019).

Elementos importantes para a composição da cultura da inovação tais como: Flexibilidade, integração, eficiência, produtividade, geração de valor, gestão e ganhos de mercado promovem uma sinergia potencializando a organização permitindo um crescimento e fomentando um ambiente favorável ao processo de inovação, eliminando alguns possíveis entraves com os envolvidos.

O processo para fomentar a culturas e a capacidade de inovação compreende a interação de vários elementos que se integram criando uma interface com várias competências. A capacidade de inovação desenvolvida por uma empresa pode ser nova para a própria empresa, nova para o setor de atividade da empresa, nova para o mercado nacional ou até mesmo para o mercado internacional (Pikkemaat \& Peters 2006; Tironi, 2011).

Sobre este ponto de vista, Tidd e Bessant (2015) estes complementam afirmando que a gestão baseada na inovação para ser adequada, deve envolver e implantar rotinas eficazes para lidar com os desafios que envolvem a inovação uma vez que diariamente o processo de gestão do conhecimento também parte integrante de todo o processo organizacional.

\subsection{Capital Humano nas Organizações}

Com o aumento da competitividade nas organizações e processo cada vez mais dinâmicos como as inovações tecnológicas, econômicas, mercadológicas e sociais encontram-se associados ao conhecimento seja este tácito ou explícito, o qual aplicado ao trabalho cria valores agregando valor e estabelecendo níveis elevados de concorrencia entre as empresas em uma escala mundial, onde o principal elemento é o capital humano utilizado nas organizações, potencializando assim o uso das ferramentas gerencias em diversas áreas da empresa.

Chiavenato (2009) afirma que as organizações não funcionam sem as pessoas e as pessoas não vivem sem as 
organizações. Ao contribuir com esta máxima o autor ratifica a importância desta relação e vai além, ao dizer que o conjunto de pessoas, talentos e competências constituem o Capital Humano das organizações.

Para outros o Capital Humano é o resultado de recursos financeiros aplicados, ou seja, o investimento realizado pelas empresas em treinamento, capacitação, retenção de valores e a capacidade de relacionamento dos seus funcionários (Wernke, Lembeck e Bronia, 2003).

De acordo com Rezende, Avilae Maia e (2012), os ativos intangíveis são divididosna competência dos funcionários, estrutura interna e estrutura externa. Dessa forma, é impossível existir uma organização sem pessoas e, mesmo estas não pertencendo ao patrimônio das empresas, são consideradas ativos, pois através de seus conhecimentos, habilidades e experiências podem desenvolver tanto ativos tangíveis quanto intangíveis.

Para que possa potencializar os resultados do capital humano alguns ementos tornan-se extremamente relevantes, fazendo com que se tenha uma relação eficiente e eficaz neste processo. Chiavenato (2004), apresenta na Figura 3 uma abordagem orientada a objetivos e estruturada em processos. Os processos são definidos com base nesses objetivos.

Figura 3: Processo de Gestão de Pessoas.

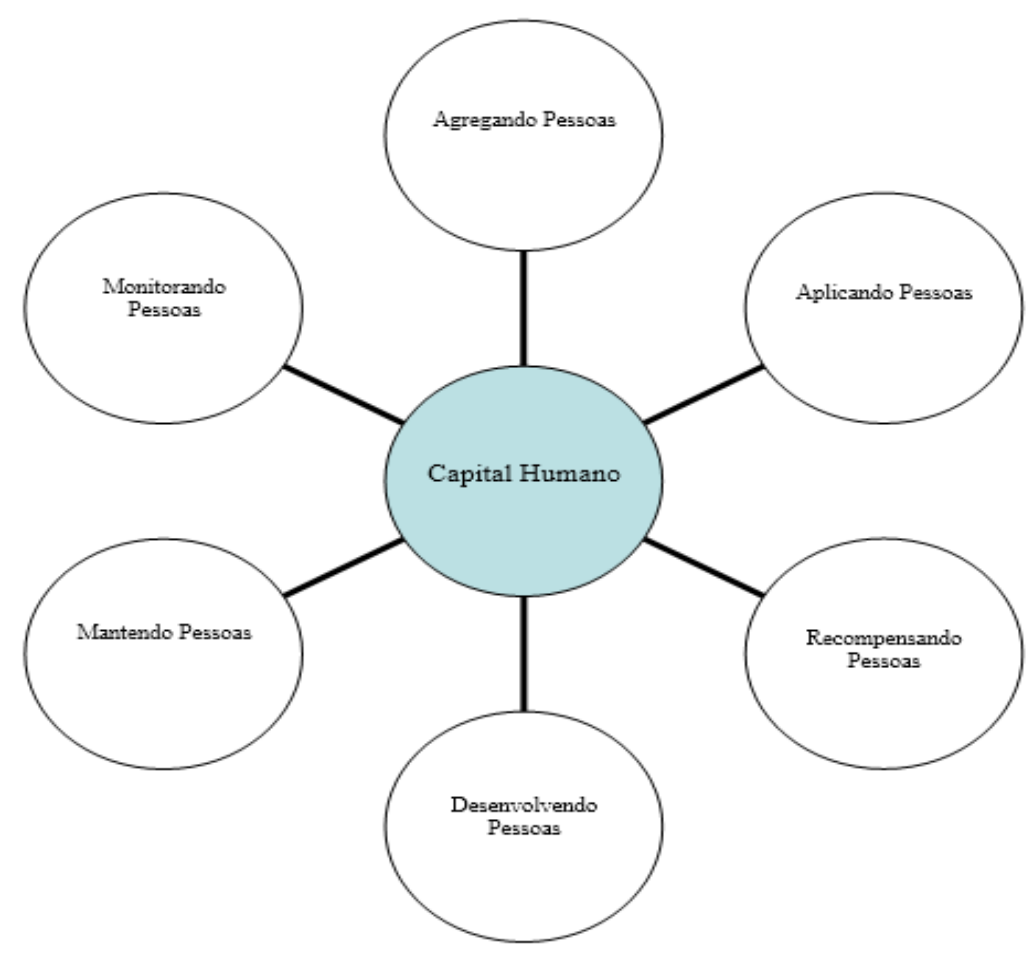

Fonte: Chiavenato (2004).

O Capital Humano nas organizações passou a ser visto com maior relevância com o avanço da globalização, fato que desencadeou uma grande mudança por parte das organizações, fazendo com que várias alterações de percepção em relação ao papel da mão de obra empregada nos vários processos no qual os trabalhadores passaram a ser considerados como colaboradores ativos no dia-a-dia da empresa, portanto, sendo também parte responsáveis pelos resultados alcançados ou não, tornando-se parte integrande deste processo. Uma das grandes preocupações que as organizações detêm hoje perante as estratégias de captação e mecanismos para a retenção de pessoas que são percebidas como parte integrante de seu ativo, esta ação passou a ser denominada atualmente como Capital Humano (Deloitte, 2017).

O capital humano está relacionado mutuamente com os capitais estrutural e relacional, pois, à medida que as pessoas e a empresa aprendem e se desenvolvem, impulsionam-se o propósito, a agilidade, o gerenciamento, a interação e o relacionamento 
com clientes, fornecedores e a outros com quem se relaciona (Neves et, al. 2020).

Marques (2018) mostra que a gestão do conhecimento aliado ao capital humamo se transformou em um instrumento essencial dentro de uma organização, uma vez que é uma maneira eficiente de usufruir a sabedoria obtida pelos colaboradores na rotina de trabalho. Este conceito por ser agregado ao exposto por Stewart (2015), que afirma que a administração do conhecimento desenvolve preceitos e métodos que tendem adquirir e compartilhar ativos intelectuais.

\subsection{Gestão Organizacional}

A disseminação e criação da informação é tão rápida e dinâmica que a maneira como esse conhecimento será gerido é de extrema importância nas organizações. As organizações podem, seguindo sua capacidade de criação de conhecimento interno e externo, avaliar as estratégias, principalmente quando ocorre troca de informações com outras organizações através de uma rede (Ferreira, 2007).

As alterações evidenciadas nos processos de gestão, dando ênfase a inovação, ao capital humamo e ao conhecimento como ativo intangível, impulsionaram as organizações à gestão contínua de seus conhecimentos em função da aprendizagem individual e coletiva de seus colaboradores (Oliveira, Castro, \& Brito, 2019).

A gestão organizacional tem se mostrado uma ferramenta de gestão de grande eficácia, sendo, portanto, imprescindível para a tomada de decisões assertivas e capazes de interferir positivamente no destino e na correta aplicação dos recursos da empresa, otimizando os resultados organizacionais (Lavarda; Pereira, 2011).

É importante salientar que as tomadas de decisões (sejam de questões simples ou complexas), para ser mais assertiva e eficientes, estas devem envolver a análise de parâmetros quantitativos e qualitativos. A se tomar decisões com base nesses critérios é comumente atribuída aos gestores dos diversos níveis hierarquicos dentro das empresas. Também deve-se levar em consideração as altas competitividades imposta pela globalização e o fator preponderante que está relacionado a lucratividade das empresas, onde é imprescindível que as decisões sejam tomadas de maneira rápida, precisa, sensata e abrangente, baseandose sempre em dados reais e concretos (Gomes; Gomes, 2012).

Desta maneira, a fim de o processo de compartilhamento de ativos tangíveis e intangíveis seja efetivo, segundo McInerney e Ali (2006), há a obrigação de que existam clima e ambiente interno que facilitem interações humanas a partir da confiança mútua, da colaboração e cooperação, do mérito a gestão organizacional centrada em regras claras que são reconhecidas, seguidas e valorizadas de forma objetiva e subjetiva para o funcionário e equipes.

Azevedo (2018) enfatiza que o ambiente que fomenta um espaço organizacional está em constante mudança, alterandose a cada geração, e esse é o motivo que faz surgir novas dinâmicas dentro de cada cenário organizacional. Essa perspectiva de constante mudança imprime uma necessidade social e organizacional que obriga as empresas públicas e privadas a desenvolverem novas fontes de recursos.

\section{Metodologia}

Neste estudo, no que se refereaos objetivos, a pesquisa possui características de referenciais teóricos. A revisão sistemática da literatura é caracterizada pela busca rigorosa de pesquisas relacionadas com seus termos de busca que seguem um protocolo e análise dos estudos localizados (Ferenhof; Fernandes, 2016).

No que diz respeito aos objetivos, trata-se de uma pesquisa descritiva. Nesse tipo de pesquisa os fenômenos (variáveis) são observados, registrados, analisados e correlacionados sem que haja manipulação dos mesmos (Cervo; Bervian; Silva, 2007).

Além disso, quanto à natureza, o presente estudo é uma investigação básica, cuja finalidade é gerar novos conhecimentos para avançar o tema "competitividade" sem aplicação prática prevista, para um problema específico. 
Segundo Marconi e Lakatos (2009), essa abordagem tem como preceito investigar e desvendar aspectos mais intrínsecos: "permitindo descrever detalhadamente investigações, atitudes e tendências de comportamento, buscando interpretar e compreender as relações acerca da complexidade do problema sem o uso de recursos estatísticos".

\section{Resultados e Discussão}

Conforme exposto no referencial teórico aliar Gestão do Conhecimento Organizacional, Inovação Organizacional, Capital Humano nas Organizações e Gestão Organizacional passam a ser um importântes fatores a serem considerados quando se trata de elementos que favoreçam as práticas relacionadas a melhoria de desempenho e em especias das empresas que estão na vangarda dos seus segmentos na visão dos autores deste artigo.

Ampliar a gestão da competitividade por meio de novas ferramentas gerenciais torna o processo desafiador, seja para quem propõe ou mesmo para aqueles que estão no processo de execução direta desta novas diretrizes e portanto podem ter algum tipo de resistência quanto ao novo. Estabelecer uma metodologia bem como esclarecer sobre como será o processo "do novo", pode ser um fator importante para a quebra de paradigmas e consequentimento um facilitador no processo de implantação desta nova visão decorrente nas organizações.

A Figura 4 apresenta segundo os autores um caminho para o entendimento de tais ações junto aos envolvidos propondo um modelo para a criação de vantagem competitiva organizacional para as organizações.

Figura 4: Modelo para criação de Vantagem Competitiva Organizacional.

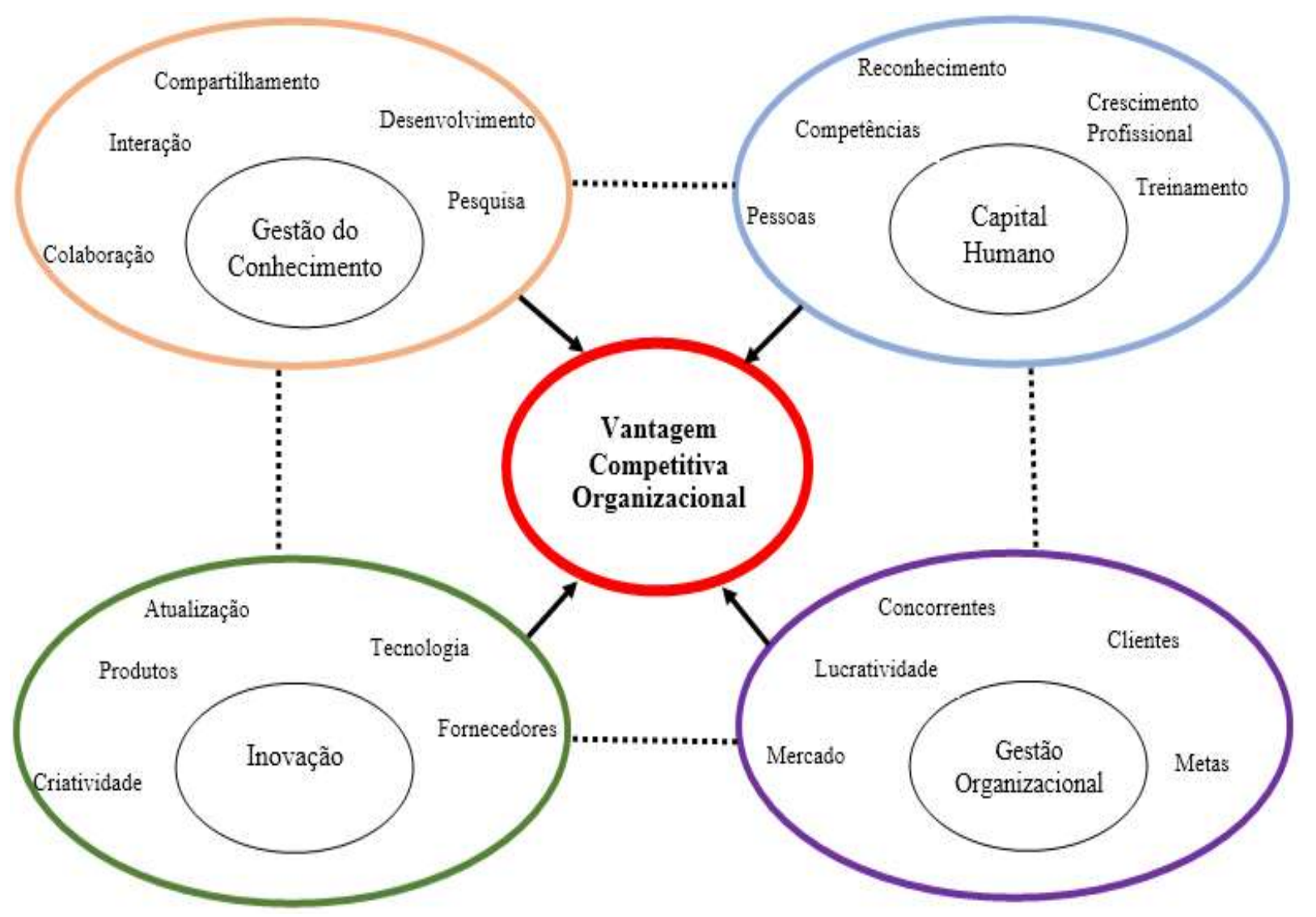

Fonte: Autores. 
Na visão dos autores a Figura 4 é uma proposta para utilização de um modelo para a obtenção de Vantagem Competitiva Organizacional onde existem algumas interfaces e interações entre alguns elementos que proporcionam este processo onde são criados subgrupos que torna possível elaborar tal modelo como:

- Gestão do Conhecimento: os sub-elementos pesquisa, compartilhamento, desenvolvimento, colaboração e interação auxiliam na formação deste item promovendo agregar valor ao conhecimento, uma vez que este é perecível e deve ser disseminado o quanto antes.

- Inovação: para estes sub-elementos tais como a tecnologia, atualização, produtos criatividade e os fornecedores fazem com que estes itens formem um processo irreversível e necessário nas organizações onde a inovação passa a ser parte integrante das empresas bem como das pessoas que estão participando direta ou indiretamente deste processo.

- Capital Humano: para este item os sub-elementos destacados são as pessoas, competências, treinamento, reconhecimento e crescimento profissional onde estes se integram e permitem a criação de mais um elemento necessário na composição desta proposta.

- $\quad$ Gestão Organizacional: Neste tópico são utilizados os sub-elementos mercado, metas, clientes, lucratividade e concorrentes onde se permite uma visão mais ampla e compartilhada das condições de mercado em que a empresa atua independentemente de seu porte ou ramo de atuação.

Com a formação destes sub-elementos são identificados os elementos que permitem a criação da vantagem competitiva organizacional possibilitando assim potencializar os resultados favoráveis para as empresas que as utilizarem na visão dos autores deste artigo, onde para que se tenha uma plena utilização do modelo proposto são fundamentais que as interfaces e interações entre os sub-elementos e os seus elementos estejam em constante atuação.

- Modelo para criação da vantagem competitiva organizacional se faz possível uma vez que os elementos gestão do conhecimento, inovação, capital humano e gestão combinadas promovem uma sinergia onde as organizações passam a utilizar o que há de melhor em suas estruturas tornando favorável a busca por melhorias sejam estas internas ou externas a organização, onde a utilização de ferramentas gerenciais já conhecidas bem como novas ferramentas são agregadores o que torna todo o processo de extrema relevância.

Não é de forma alguma e nem pretensão dos autores esgotarem esta temática e sim promover e fomentar tal discussão sobre o tema onde as organizações devem sempre estar em um processo de inovação e melhoria contínua de novos modelos para alcançarem sua maior eficiência e eficácia tanto operacional quanto organizacional por meio de tais metodologias.

\section{Conclusão}

O objetivo principal deste trabalho foi apresentar um modelo teórico para vantagem competitiva organizacional utilizando quatro elementos e seus sub-elementos: Gestão do Conhecimento, Inovação, Capital Humano e Gestão Organizacional agregando volor e potencializando as ações a serem realizadas pelas empresas.

Com isso pretende-se ampliar a performance bem como as tomadas de decisões estratégicas fazendo com que as organizações possam obter um melhor desempenho e consequentimento uma maior lucratividade. Deve-se levar em conta a questão do envolvimento das pessoas em todos os processos, sejam eles administrativos e/ou produtivos. 
Como contribuições do artigo realizada, buscou-se avançar nas discussões sobre as vantagens competitivas nas organizações por meio de uma nova proposta de modelo que deve aplicado na pratica em qualquer tipo de empresa para que se possa ter sua validação, e deixando aqui também como pesquisa futura a comparação de tal modelo em empresas de diferentes ramos e atuações.

Ampliar a base para novas discussões é o intuito dos autores que buscam estabelecer novas metodologias bem como novas aplicações. Com isso na visão dos autores, pode-se contribuir de fato com os setores e estratégias que podem ser internas, externas (entidades setoriais) ou ainda em políticas de desenvolvimento que devem gerar impacto e agregar valor as empresas e as pessoas envolvidas.

Estimulas a pesquisa e desenvolvimento deve ser ponto central de qualquer pesquisa, independentemente dos resultados serem positivos ou negativos, o aprimoramento de tais ações deve ser ponto relevante quanto se trata de uma contribuiçãoacadêmica empresarial ou até mesmo socio-ambiental.

É de interesse dos autores ainda, a partir da discussão em fóruns especializados em Gestão da Inovação, Gestão do Conhecimento, Gestão de Pessoas e de Desenvolvimento, aproximar o esta discussão entre diversos pesquisadores e diferentes áreas mostrando assim a importância da interação e interface na geração de novas idéias e tecnologias, permitindo assim consequentemente uma melhora na performance organizacional.

\subsection{Trabalhos futuros}

$\checkmark$ Aplicação do modelo proposto e analisar quais os seus resultados nas organizações.

\section{Agradecimentos}

$\checkmark$ Aos nossos familiares pelo apoio, carinho e compreensão.

\section{Referências}

Abualoush, S. H., Obeidat, A. M., Tarhini, A., \& Al-Badi, A. (2018). O papel da capacitação dos funcionários como variável intermediária entre a gestão do conhecimento e os sistemas de informação sobre o desempenho dos funcionários. VINE Journal of Information and Knowledge Management Systems.

Altman, D., Burton, N., Cuthill, I., Festing, M., Hutton, J., \& Playle, L. (2006). Why do a pilot study. National Centre for Replacement, Refinement and Reduction of Animal in Research, 12.

Bervian, P. (2007). CERVO. AL. SILVA, R. da. Metodologia Científica. (6a ed.), Pearson Prentice Hall.

Bessant, J., Tidd, J., \& Pavitt, K. (2008). Gestão da inovação. Porto Alegre, 3.

Boly, V., Morel, L., \& Camargo, M. (2014). Evaluating innovative processes in french firms: Methodological proposition for firm innovation capacity evaluation. Research Policy, 43(3), 608-622.

Carrincazeaux, C., \& Gaschet, F. (2015). Regional innovation systems and economic performance: Between regions and nations. European Planning Studies, 23(2), 262-291.

Chiavenato, I. (2003). Administração de recursos humanos: fundamentos básicos. Atlas.

Chiavenato, I. (2010). Gestão de pessoas.

Chuang, S. H., Liao, C., \& Lin, S. (2013). Determinants of knowledge management with information technology support impact on firm performance. Information Technology and Management, 14(3), 217-230.

da Rosa, M. P. D. S., Neves, M. S., \& de Sá Freire, P. (2020, November). O Retorno Do Investimento Na Gestão Do Conhecimento Com Foco No Capital Humano. In Anais do Congresso Internacional de Conhecimento e Inovação-ciki (Vol. 1, No. 1).

Dávila, G. A., Durst, S., \& Varvakis, G. (2018). Knowledge absorptive capacity, innovation, and firm's performance: insights from the South of Brazil. In Managing Knowledge, Absorptive Capacity and Innovation (pp. 475-508).

Davila, T., Epstein, M., \& Shelton, R. (2012). Fazendo a inovação funcionar: como gerenciá-la, medi-la e lucrar com ela . Pressione FT.

de Abreu, P. H. C. (2018). Perspectivas para a Gestão do Conhecimento no Contexto da Indústria 4.0. South American Development Society Journal, 4(10), 126145 . 
de Carvalho Rezende, J. F., Avila, M., \& Maia, R. S. (2012). Geração e gestão do valor por meio de métricas baseadas nas perspectivas do capital intelectual. Revista de Administração, 47(1), 51-67.

de Mendonça Azevedo, I. (2018). Análise e aplicação bibliométrica na administração e áreas afins: um levantamento nacional. Revista InterScientia, 6(2), 146164.

de Oliveira Morais, M., Brejão, A. S., \& Neto, P. L. D. O. C. Elementos para uma inovação organizacional: Estudo de caso em uma empresa metalúrgica.

de Pina Lopes, E., \& Chaves, M. C. C. (2012). Carlos Francisco Simões Gomes Universidade Federal Fluminense \& Ibmec-RJ carlos. francisco@ pq. cnpq. br Helder Costa Universidade Federal Fluminense.

de Sá, F. B., dos Reis Bento, K. G., Ziviani, F., \& Ferreira, M. A. T. (2013). Práticas de gestão do conhecimento: um estudo em organizações mineiras. Perspectivas em Gestão \& Conhecimento, 3(1), 114-131.

Fagerberg, J., \& Sapprasert, K. (2011). Sistemas nacionais de inovação: o surgimento de uma nova abordagem. Ciência e políticas públicas, 38 (9), 669-679.

Ferenhof, H. A., \& Fernandes, R. F. Desmistificando a revisão de literatura como base para redação científica: método SFF Demystifying The Literature Review As Basis For Scientific Writing: SSF METHOD. Revista ACB; v. 21, n. 3 (2016): Anais do 34 Painel Biblioteconomia em Santa Catarina (2016); 550$563,24(2), 563-550$.

Ferreira, V. R. B. (2007). A utilização de práticas de gestão do conhecimento em organizações da sociedade civil que trabalham com projetos de inclusão digital: uma estudo de caso.

Franco, J. B. M., Formiga, N. S., Grangeiro, S. R. A., Oliveira, H. C. C., \& Estevam, I. D. (2021). Correlatos E Variações Na Percepção Do Suporte Organizacional E Gestão Do Conhecimento Em Trabalhadores De Organizações Públicas E Privadas Em Natal-RN. RECIMA21-Revista Científica Multidisciplinar-ISSN 2675-6218, 2(4), e24213-e24213.

Greening, D. W., \& Turban, D. B. (2000). Corporate social performance as a competitive advantage in attracting a quality workforce. Business \& society, 39(3), 254-280.

Jannuzzi, C. S. C., Falsarella, O. M., \& Sugahara, C. R. (2016). Gestão do conhecimento: um estudo de modelos e sua relação com a inovação nas organizações. Perspectivas em Ciência da Informação, 21, 97-118.

Karlsson, M. P. (2010). Collaborative idea management.

Lavarda, C. E. F., \& Pereira, A. M. (2011). Planejamento e controle orçamentário empresarial como ferramenta de apoio à tomada de decisão. ABCustos, 6(1).

Marconi, M. D. A., \& Lakatos, E. M. (2009). Fundamentos de metodologia cientifica. Atlas.

Marques, J. R. (2018). A relação entre a gestão do conhecimento e o capital intelectual nas organizações. Portal IBC.

Menezes, R. G., \& De Muylder, C. F. (2020). Inteligência competitiva, inovação e performance: proposta de modelo teórico. Brazilian Journal of Development, 6(1), 657-678.

Mothe, C., \& Thi, T. U. N. (2010). The link between non-technological innovations and technological innovation. European Journal of Innovation Management.

Neves, M. L. C., Marcon, G. A., Trzeciak, D. S., \& Neves, E. R. (2018). Gestão do conhecimento no brasil: A abordagem dos textos jornalíticos. Perspectivas em Gestão \& Conhecimento, 8(3), 228-242.

Nonaka, I., \& Takeuchi, H. (1995). The knowledge-creating company: How Japanese companies create the dynamics of innovation. Oxford university press.

Okumus, F. (2013). Facilitating knowledge management through information technology in hospitality organizations. Journal of Hospitality and Tourism Technology.

Oliveira, Z. M. F. D. (2010). Fatores influentes no desenvolvimento do potencial criativo. Estudos de psicologia (Campinas), 27, 83-92.

Pereira, R. (2021). Gestão do Conhecimento aliada ao crescimento organizacional: perspectivas à prática hospitalar. AtoZ: novas práticas em informação e conhecimento, 10(1), 103-112.

Pikkemaat, B., \& Peters, M. (2006). Informações de mercado: um fator chave de sucesso no processo de desenvolvimento de novos produtos no turismo. Erlebnisinszenierung im Tourismus: Erfolgreich mit emocionalen Produkten und Dienstleistungen, 81-96.

Polanyi, M. (1966). The tacit dimension. Double Day \& Co. Garden City.

Ramos, N. K., Yamaguchi, C. K., \& da Costa, U. M. (2020). Tecnologia da informação e gestão do conhecimento: estratégia de competitividade nas organizações. Brazilian Journal of Development, 6(1), 144-161.

Schein, E. H. (2017). Cultura organizacional e liderança. Atlas.

Schubert, T. (2010). Marketing and organisational innovations in entrepreneurial innovation processes and their relation to market structure and firm characteristics. Review of Industrial Organization, 36(2), 189-212.

Schumpeter, J. A. T. D. D. (1982). ECONÔMICO. Abril Cultural.

Silva, B. E., de Ramos Neves, J. T., Vasconcelos, M. C. R. L., \& Araújo, C. A. Á. (2013). Contribuições da inovação aberta para uma empresa de comunicação. Revista Gestão \& Tecnologia, 13(2), 222-246. 
Research, Society and Development, v. 10, n. 9, e44310918388, 2021 (CC BY 4.0) | ISSN 2525-3409 | DOI: http://dx.doi.org/10.33448/rsd-v10i9.18388

Souza, E. S. D. (2018). Características e impactos da indústria 4.0: percepção de estudantes de ciências contábeis.

Souza, J. C., \& de Fátima Bruno-Faria, M. (2013). Processo de inovação no contexto organizacional: uma análise de facilitadores e dificultadores. BBR-Brazilian Business Review, 10(3), 113-136.

Terra, J. (2012). Dez mandamentos da gestão da inovação: uma abordagem para a transformação organizacional.

Tidd, J., \& Bessant, J. (2015). Gestão da inovação-5. Bookman Editora.

Tironi, E., \& Cavallo, A. (2011). Comunicación estratégica. Vivir en un mundo de señales. Taurus.

Wernke, R., Lembeck, M., \& Bornia, A. C. (2003). As considerações e comentários acerca do capital intelectual. Revista da FAE, 6(1). 